Network Working Group

Request for Comments: 1609

Category: Experimental
G. Mansfield

AIC Systems Laboratory

$\mathrm{T}$. Johannsen

Dresden University

M. Knopper

Merit Networks, Inc.

March 1994

\title{
Charting Networks in the X.500 Directory
}

Status of this Memo

This memo defines an Experimental Protocol for the Internet community. This memo does not specify an Internet standard of any kind. Discussion and suggestions for improvement are requested. Distribution of this memo is unlimited.

Abstract

There is a need for a framework wherein the infrastructural and service related information about communication networks can be made accessible from all places and at all times in a reasonably efficient manner and with reasonable accuracy. This document presents a model in which a communication network with all its related details and descriptions can be represented in the X.500 Directory. Schemas of objects and their attributes which may be used for this purpose are presented. The model envisages physical objects and several logical abstractions of the physical objects. 
Table of Contents

1. Introduction 2

2. Infrastructural information requirements 2

3. The Nature of the Network Map - The X.500 Solution 4

4. The hierarchical model of a network 5

4.1 Network maps 5

4.2 Representation in the X.500 Directory 6

5. Position in The Directory Information Tree(DIT) 7

6. Proposed Schemes 8

6.1 Communication Object Classes 9

6.2 Physical elements 10

6.2 .1 Network 10

6.2.2 Node 11

6.2.3 NetworkInterface 12

6.3 Logical Elements 12

6.3.1 Network 13

6.3 .2 Node 13

6.3.3 NetworkInterface 13

7. Security Considerations 14

8. Authors' Addresses 14

9. References 15

1. Introduction

The rapid and widespread use of computer networking has highlighted the importance of holding and servicing information about the networking infrastructure itself. The growing and active interest in network management, which has concentrated mainly in the areas of fault and performance management on a local scale, is severely constrained by the lack of any organized pool of information about the network infrastructure itself. Some attempts have been made, on a piecemeal basis, to provide a larger view of some particular aspect of the network (WHOIS, DNS, .. in the case of the Internet; [1], [2]). But to date, little or no effort has been made in setting up the infrastructural framework, for such an information pool. In this work we explore the possibility of setting up a framework to hold and serve the infrastructural information of a network.

2. Infrastructural information requirements

Network operation and management requires information about the structure of the network, the nodes, links and their properties. Further, with current networks extending literally beyond bounds, the scope of the information covers networks beyond the span of local domain of authority or administration. When the Network was relatively small and simple the map was already known to the knowledgable network administrator. Based on this knowledge the 
course of the packets to different destinations would be charted. But presently the size of the Network is already beyond such usages. The current growth of the Network is near explosive. This is giving rise to the urgent necessity of having infrastructural and service related information made accessible from all places and at all times in a reasonably efficient manner and with reasonable accuracy. In the rest of this work a network is the media for transmitting information. Network elements are equipment with one or more network interfaces whereby it is possible to exchange information with the network. Network elements with multiple interfaces e.g., gateways/routers/bridges/repeaters... may be used to connect networks. Network related information, referred to as 'network map' in the rest of this paper, should

1. Show the interconnection between the various network elements. This will basically represent the Network as a graph where vertices represent objects like gateways/workstations/ subnetworks and edges indicate the connections.

2. Show properties and functions of the various network elements and the interconnections. Attributes of vertices will represent various properties of the objects e.g., speed, charge, protocol, os, etc. Functions include services offered by a network element.

3. Contain various name and address information of the networks and network elements

4. Contain information about various administrative and management details related to the networks and network elements.

5. Contain the policy related information, part of which may be private while the other part may be made public.

Using this map the following services may be provided

1. Configuration management:

- Display the physical configuration of a network, i.e., nodes and their physical interconnections

- Display the logical configuration of a network, i.e., nodes and their logical interconnections.

2. Route management:

- Find alternate routes by referring to the physical and logical configurations.

- Generate routing tables considering local policy and policy of transit domains 
- Check routing tables for routing loops, non-optimality, incorrect paths, etc.

3. Fault management: In case of network failures alternatives may be found and used to bypass the problem node or link.

4. Service management: Locate various services and servers in the Network.

5. Optimization: The information available can be used to carry out various optimizations, for example cost, traffic, response-time, etc.

6. Provide mappings between the various names and addresses of elements

7. Depict administrative/autonomous domains.

8. Network Administration and Management: References to people responsible for administering and technically maintaining a network will be useful.

Examples of such usages are described in [3], [4].

3. The Nature of the Network Map - The X.500 solution

Implementing and maintaining a detailed map of the network poses a serious problem. The scope of the map is global and the network itself is expanding. Some of the problems that are peculiar to the network map are listed below.

- The Network configuration is quasi-static. Nodes, links and networks are being added, updated and deleted someplace or the other.

o The Network is huge and geographically distributed.

- The network spans several political and administrative areas. The related information is also controlled and maintained in a distributed fashion.

In short, global network configuration information is unwieldy and growing continuously. It is impossible to service such information in a centralized fashion. There is need for a distributed framework which allows users and applications to access information about users, services, networks, ... easily and globally. The OSI X.500 Directory services [5] provides a rich framework to support a 
globally distributed information service system. The X.500 Directory is intended to be a very large and highly distributed database. It is structured hierarchically with entries arranged in the form of a tree in which each object corresponds to a node or an entry. Information is stored about an object as a set of attributes.

4. The hierarchical model of a network

For representing networks in the Directory we use the following hierarchical model.

A network is the media for transmitting information with zero or more network elements each having at least one network interface on the media. The media may be any kind of a line (physical circuit/virtual circuit), or a collection of interconnected networks.

$<$ The postscript version of this document >

$<$ has a figure here. However, the figure >

<is too complex to be drawn in simple ASCII.>

Figure 1: Simple and composite networks and their mapping to the DIT.

The model allows hierarchy of subnetworks. Network elements with multiple interfaces may act as external gateways to the attached network and to networks higher up in the hierarchy. Thus, a gateway may be the external gateway of several networks which are either interconnected or have a hierarchical relationship.

A network may be simple consisting of zero or more network elements or composite consisting of several sub-networks. Examples of simple networks are ethernets, optical fiber/copper cables, free space, .. .

\subsection{Network Maps}

Using the above model it is straight forward to draw the topological graph of the network where the vertices represent the components of the network and edges indicate the connections. For visual representation the graph may be translated to a more "physical" illustration (figure 1).

Just as there are several maps of the same geographical domain (political, natural...) one can envisage several views of the same network and its components. A view (called "image" in the remainder) could pertain to a particular protocol suite (IP/OSI/...), an administrative domain or purpose. Using images, several abstractions of the same object are possible. 


\subsection{Representation in the X.500 Directory}

To represent the various images of networks and its components along with the real-image relationship among the various objects we introduce the following classes of objects:

- Communication Object Class (CO): All objects defined furtheron in this document belong to this class. Common attributes for all communication objects are defined in section 6 .

- Physical Communication Object Class (PCO): A subclass of CO-class, this class defines common properties for all objects representing physical communication objects.

- Image Communication Object Class (ICO): A subclass of co-class, this class defines common properties for all objects representing images of communication objects.

The above classes sort communication objects into physical or image object. As is implied in the nomenclature a physical object will have several attributes describing it physical properties - location, weight, size, .... etc. An image object will have an Image-of attribute. The Image-of attribute will point to a physical object or to another image object.

Using this schema it is possible to represent the case of several logical network systems (running different protocol stacks - IP, XNS, SNA, OSI, ...) which coexist on the same physical network. Information related to different types of networks, no matter what the underlying communication protocol is, will reside in the Directory in harmony. Also, their interrelation will be represented and accessed in a fashion independent of the source/destination network, namely, using the OSI X.500 protocol.

Schemes for physical networks and logical images of physical networks are defined in section 6 .

All objects are defined in section 6 . 


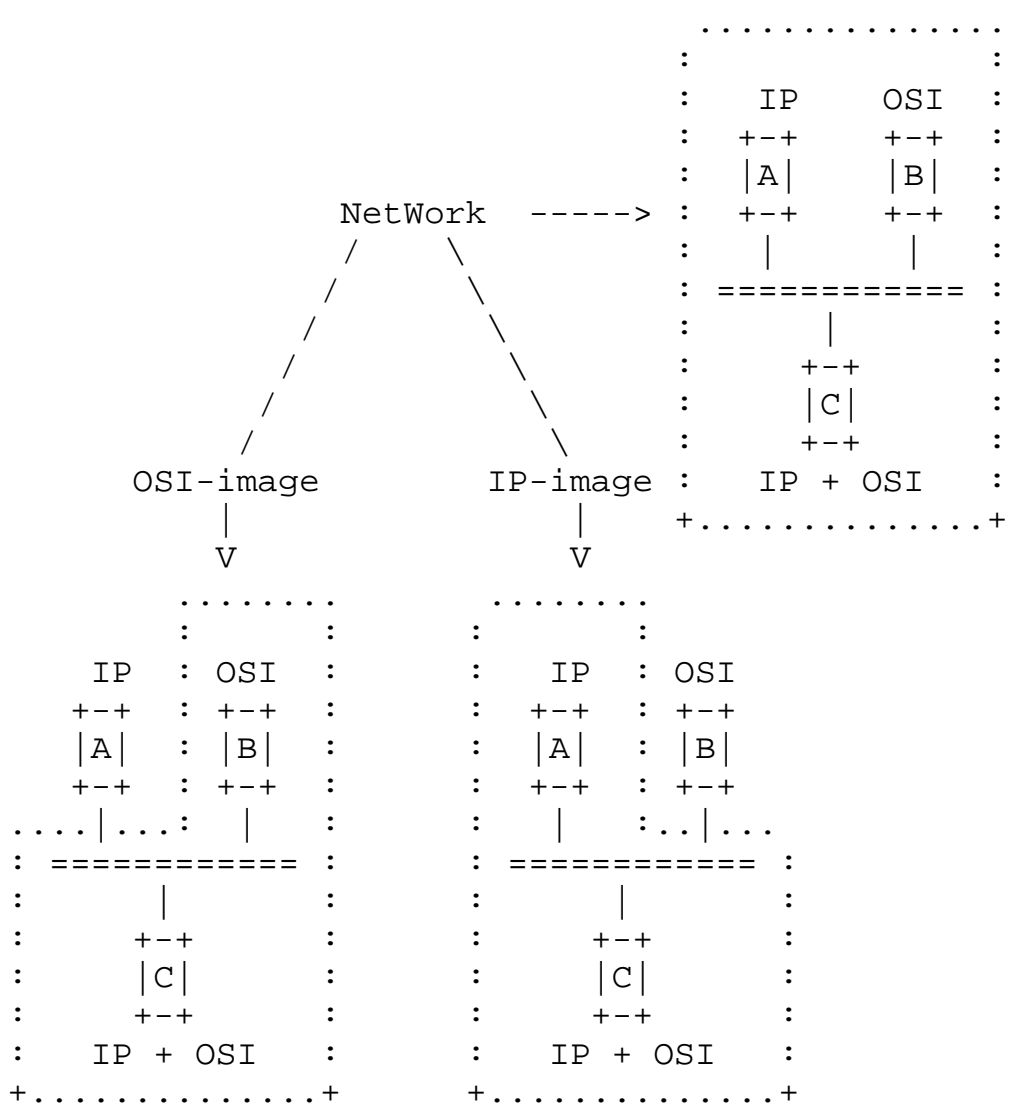

Figure 2: Several logical views of the same physical network

5. Position in the Directory Information Tree (DIT)

Information about networks usually will be contained in the DIT as subordinate of the organization maintaining the network. The network model gets mapped into a tree structure for network elements. There is one network object giving general descriptions of the network. Subordinates of this network object are node objects for each node element present in the network. Node objects hold networkInterface objects as subordinates. A network can be physically or logically subdivided into several (sub)networks. In this case, a network entry will have network objects as subordinates which again build the same structure. These entries may be kept as subordinates of organizationalUnit entries as well, with pointers from the "root" network. 
This structure holds for physical and logical elements. Physical elements are named network, node and networkInterface, and logical elements are named networkImage, nodelmage and networkInterfaceImage.

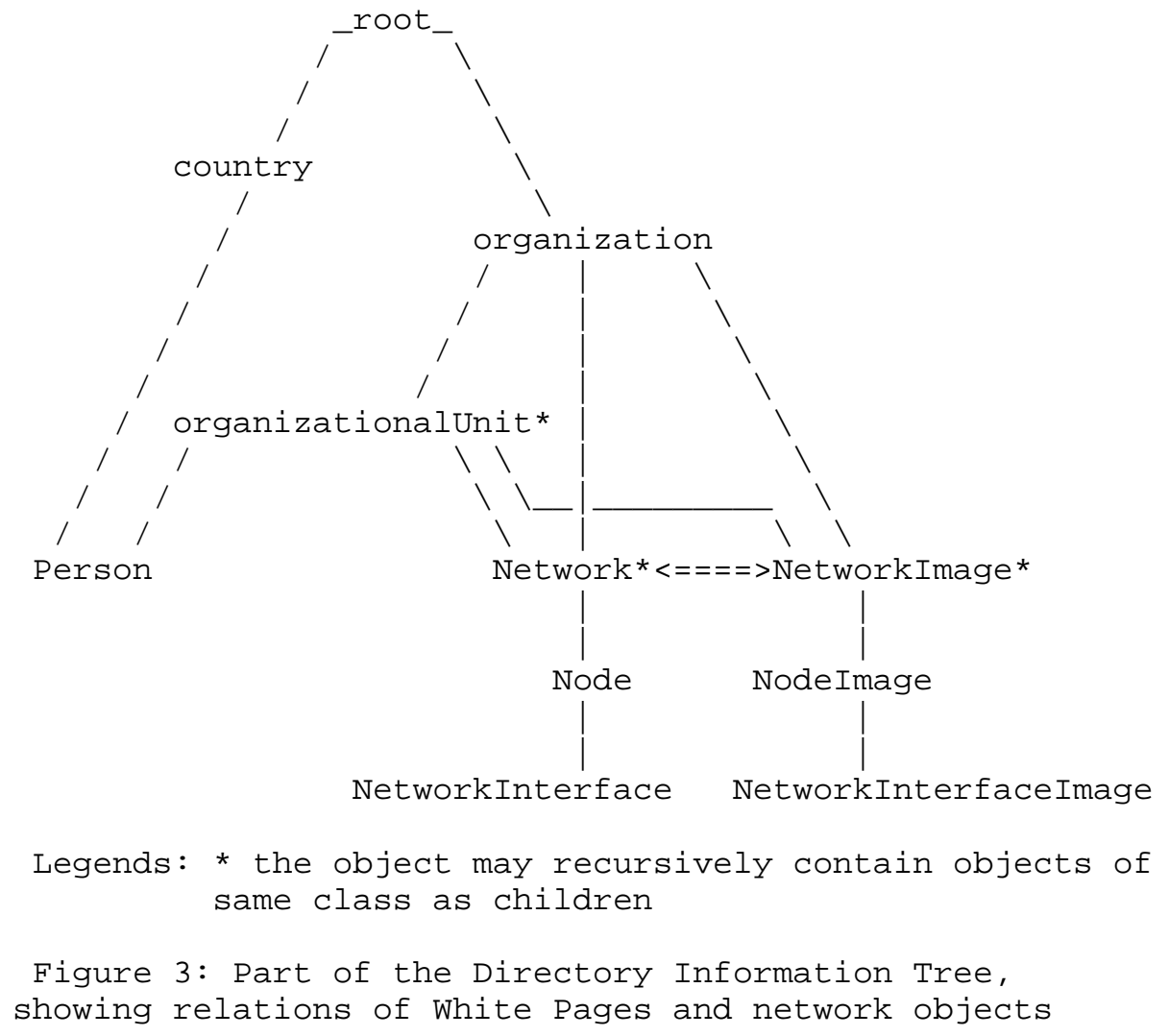

6. Proposed Schemes

A physical network comprises of wires and machines. The physical map of the network will show the interconnections of these nodes by these circuits.

For each physical network element, one or more images may exist. Similarly, an image may be attached to one or more physical objects. The types of images can grow along with the requirements. Relationship between elements (physical or logical) are expressed by attributes or the position in the Directory tree. 
Problems that are addressed in the schema:

1. Avoiding data duplication

2. Preserving administrative boundaries/controls.

3. Simple representation (minimal number of pointers)

4. Security: Though no special emphasis has been placed in this work we believe the X.500 access control policies policies will provide a reasonable secure framework for security and privacy.

Problems that are not addressed:

1. Caching policies, etc.: to be decided locally

6.1 Communication Object Classes

The object classes introduced in section 4 are defined as follows:

Communicationobject OBJECT CLASS

SUBCLASS of top

MAY CONTAIN \{

description : : CaseIgnorestringSyntax,

/* can contain any information about the object, however, wherever an appropriate attribute exists, this should be used first to hold information */

adminContact : : distinguishedNamesyntax,

/* points to the person which is responsible for the administration of the instance this object describes;

This refers to the instance only in the context of the concrete object class * /

techncontact :: distinguishedNamesyntax,

I* points to the person which is responsible for the technical maintenance of the instance this object describes;

This refers to the instance only in the context of the concrete object class;

Availability (e.g. hours of service) is not

\} covered by this attribute. */

PhysicalCommunicationobject OBJECT CLASS

SUBCLASS of Communicationobject

MAY CONTAIN \{

owner : : distinguishedNamesyntax,

/* refers to organization or person owning the physical element; 


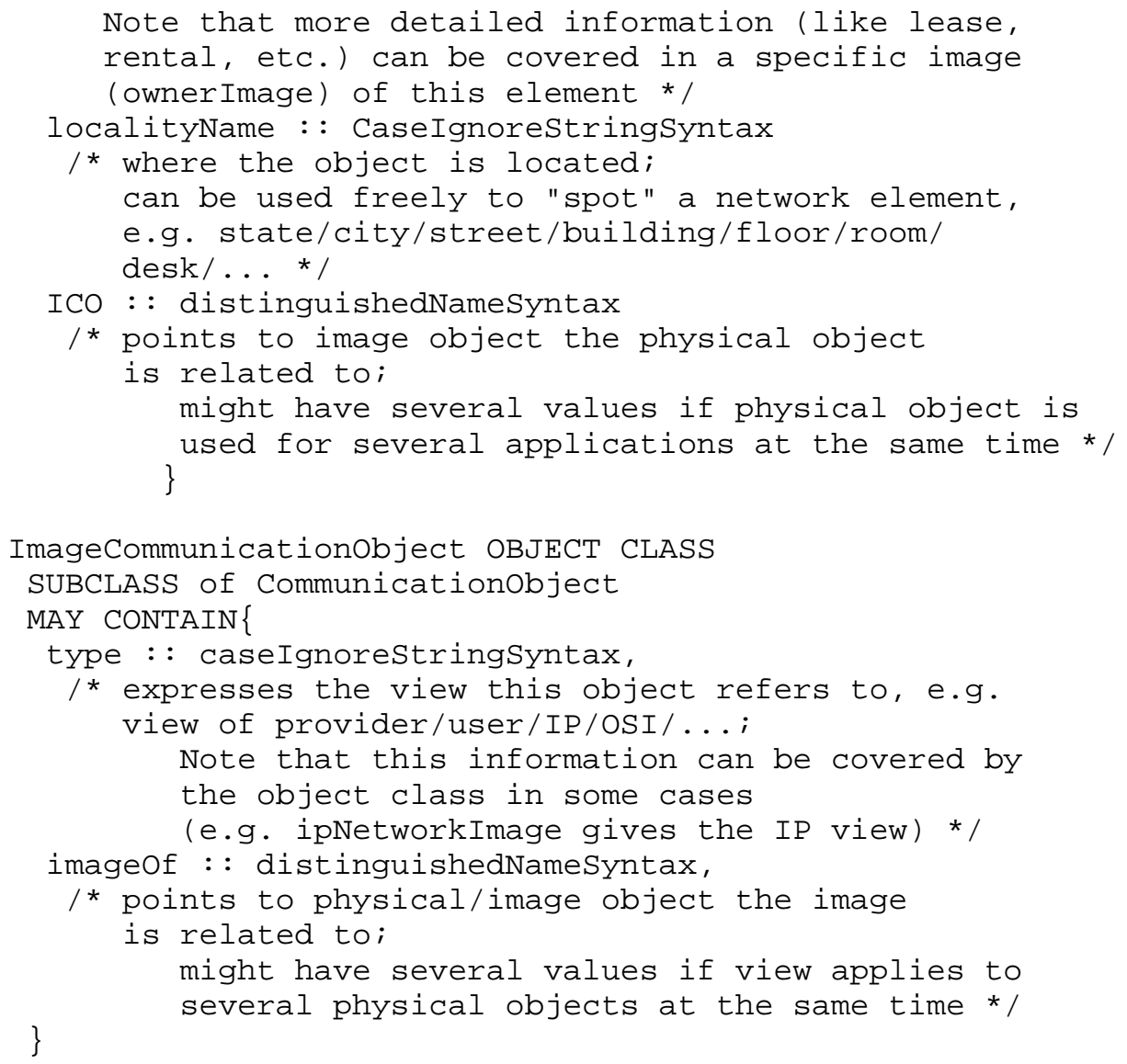

6.2 Physical elements

The following objects describe network elements without saying anything about their usage. All objects inherit properties of the Physicalcommunicationobject class.

\subsubsection{Network}

The network object supplies general descriptions which are common for a set of nodes and circuits comprising one network. This includes information about the type of circuits (medium, broadcast or pointto- point, etc.) and properties (speed etc.).

network OBJECT CLASS SUBCLASS of PhysicalCommunicationobject MUST CONTAIN \{ networkName : : caseIgnoreStringSyntax \} 


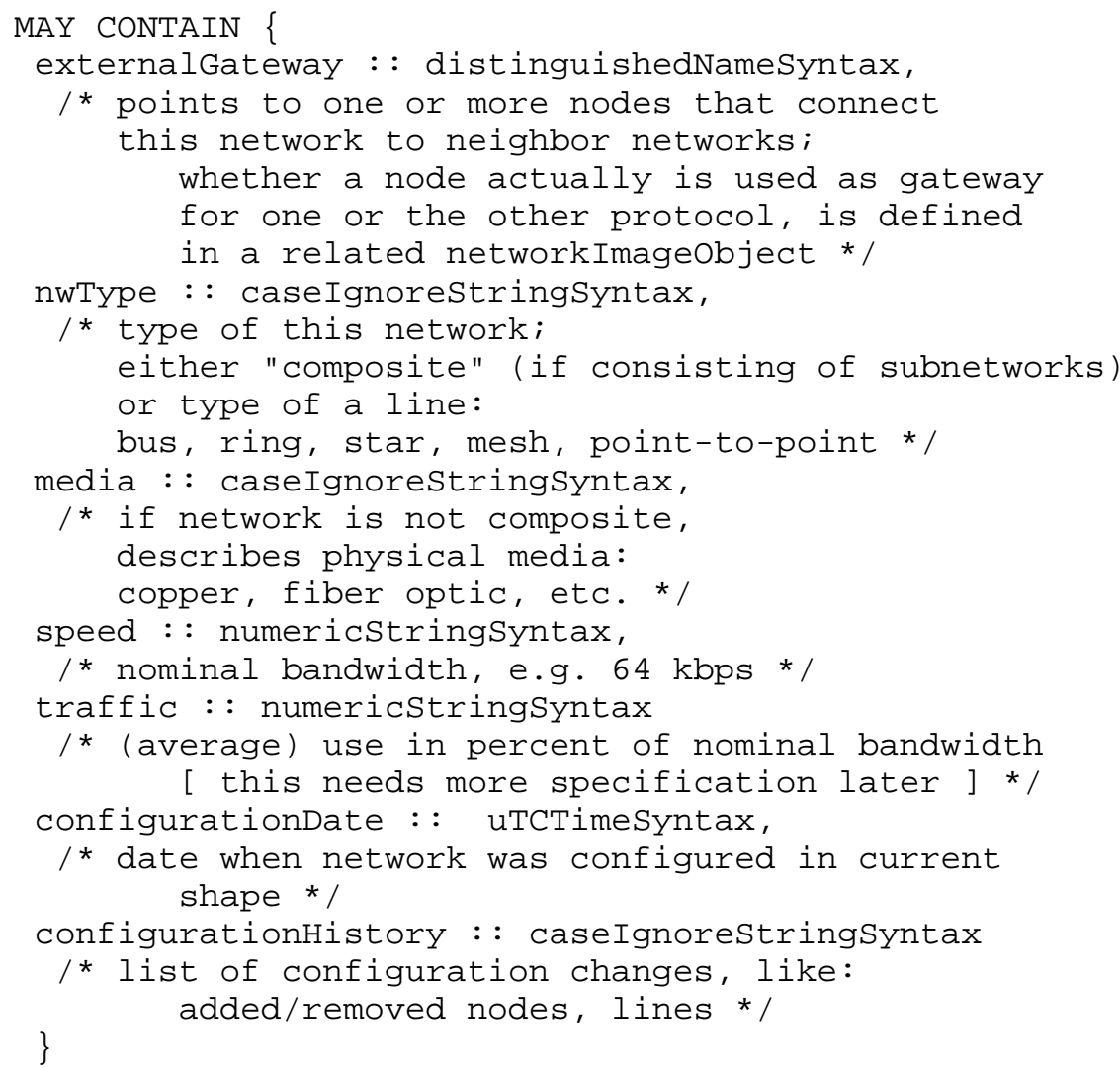

6.2 .2 Node

The node object describes any kind of device that is part of the network, such as simple nodes, printer, bridges.

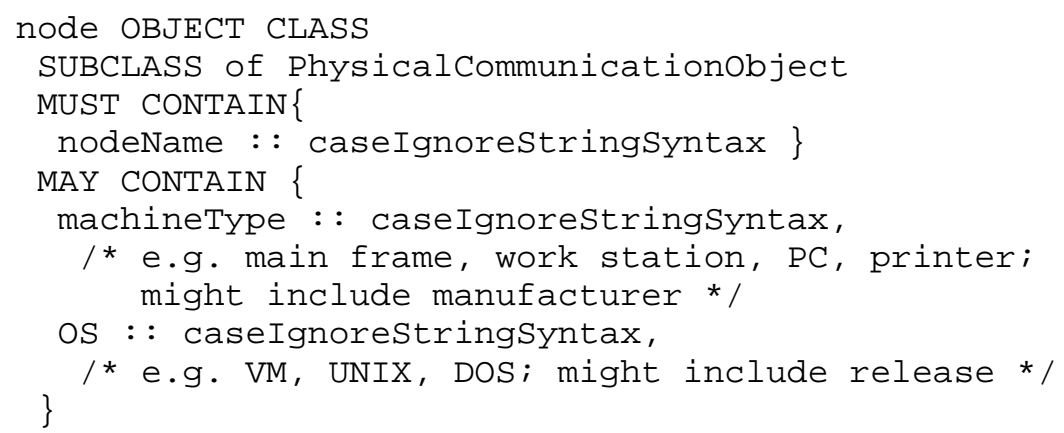




\subsubsection{NetworkInterface}

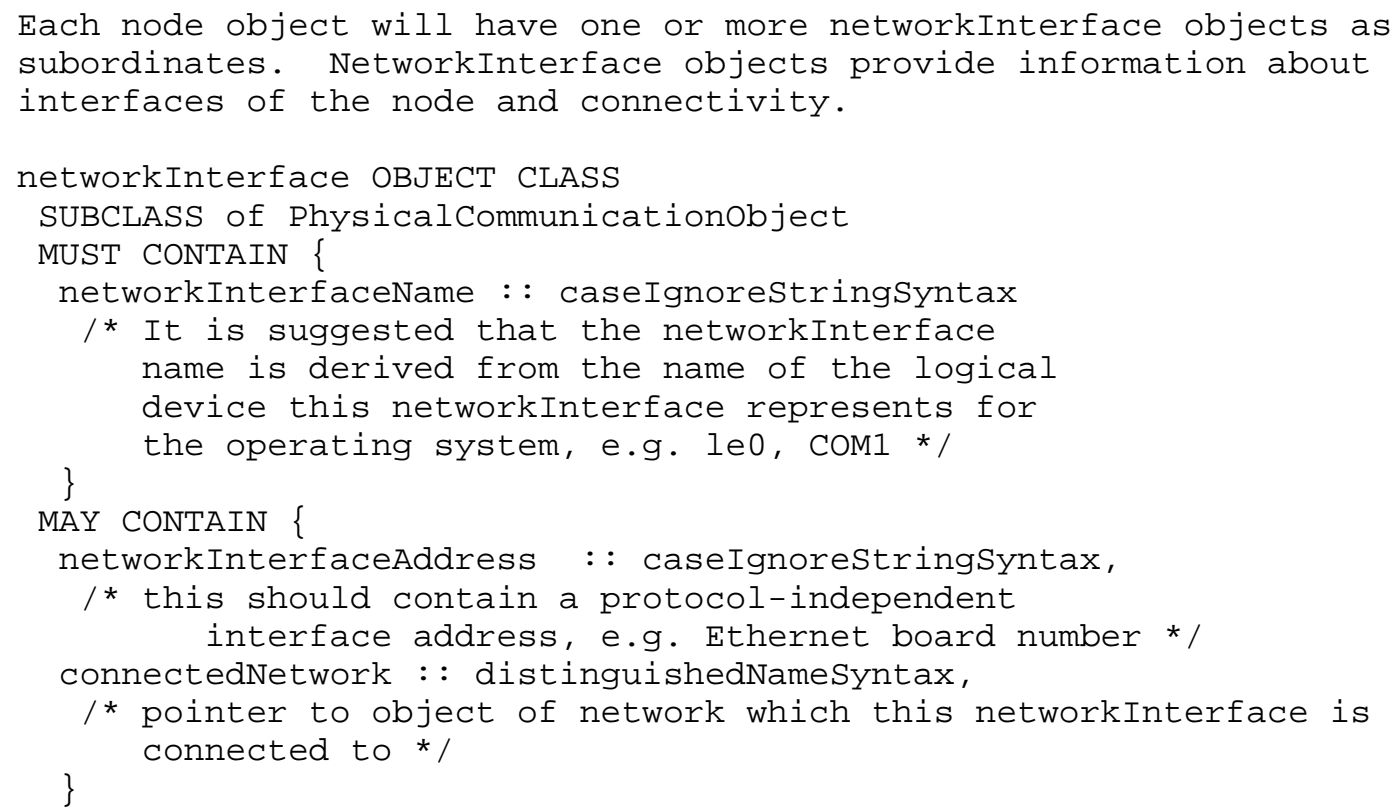

\subsection{Logical Elements}

An abstract view of a physical element is called image in this document. The word image gets appended to the object type, leading to the new objects networkImage, nodeImage and networkInterfaceImage. Images will either build Directory trees of themselves or be stored as part of the physical network tree (see section 5).

Image objects inherit properties of the ImageCommunicationobject class.

Each image object has specific attributes which vary depending on the point of view (IP, OSI, ....). Also, the user and provider of an image will view an object differently; further a user of an object may also be providing the services of the same object to another user.

Therefore, in the following a complete and general list of attributes cannot be given. We recommend to define subclasses of Image classes for each logical view. These subclasses inherit all attributes defined with the object classes below and add more specific attributes. Examples for an IP-view are given in [1]. 


\section{3 .1 Network}

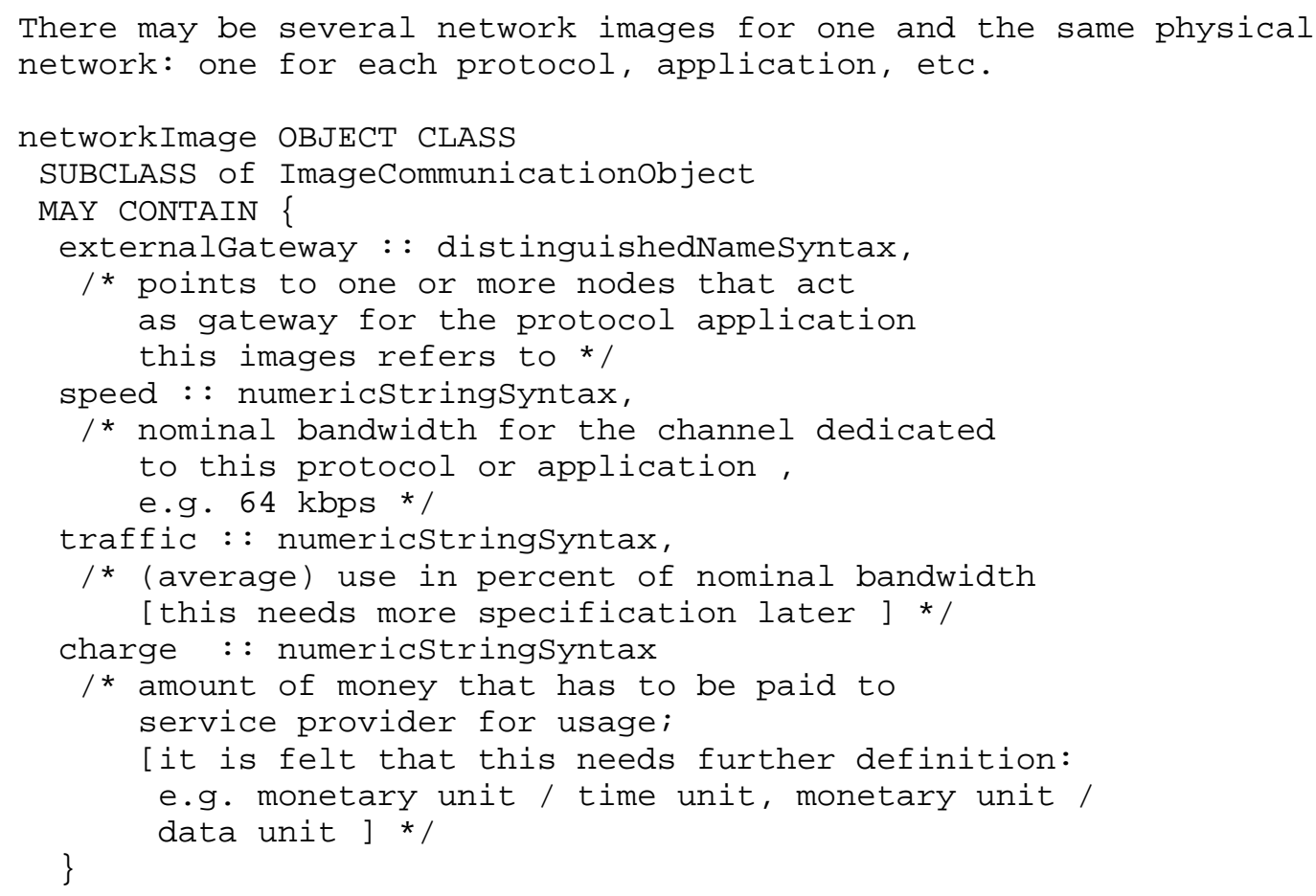

\section{3 .2 Node}

Name and functionality within the network might vary for a node from protocol to protocol considered. In particular, a node might act as gateway for one protocol but not for the other. Routing policy is stored in the case of policy gateways.

nodeImage OBJECT CLASS

SUBCLASS of ImageCommunicationobject

/* no attributes common for all nodelmages have been defined yet */

\section{3 .3 NetworkInterface}

As with physical nodes, nodelmages have networkInterfaces (networkInterfaceImages) which describe connectivity to other network elements. NetworkInterfacelmages are only given if the protocol is establishing connections via this networkInterface.

networkInterfaceImage OBJECT CLASS

SUBCLASS of ImageCommunicationobject 


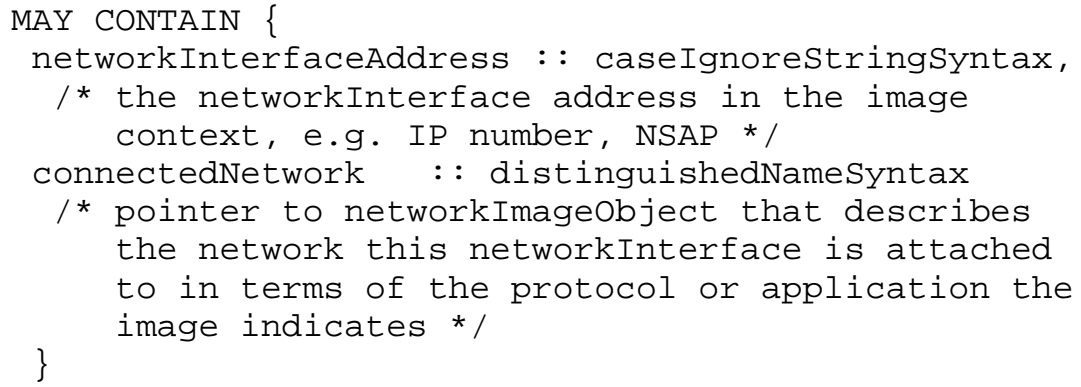

7. Security Considerations

Security issues are not discussed in this memo.

8. Authors' Addresses

Glenn Mansfield

AIC Systems Laboratory

6-6-3 Minami Yoshinari

Aoba-ku, Sendai 989-32

Japan

Phone: +81 22 279-3310

EMail: glenn@aic.co.jp

Thomas Johannsen

Dresden University of Technology

Institute of

Communication Technology

D-01062 Dresden

Germany

Phone: +49351 463-4621

EMail: Thomas.Johannsen@ifn.et.tu-dresden.de

Mark Knopper

Merit Network, Inc.

1071 Beal Avenue

Ann Arbor, MI 48109

EMail: mak@merit.edu 
9. References

[1] Harrenstein, K., Stahl, M., and E. Feinler, "NICNAME/WHOIS", RFC 954, SRI, October 1985.

[2] Mockapetris, P., "Domain Names - Implementation and Specification", STD 13, RFC 1035, USC/Information Sciences Institute, November 1987.

[3] Johannsen, T., Mansfield, G., Kosters, M., and S. Sataluri, "Representing IP information in the X.500 Directory", RFC 1609, Dresden University, AIC Systems Laboratory, Network Solutions, Inc., AT\&T Bell Laboratories, March 1994.

[4] Johannsen, T., and G. Mansfield, "The Soft Pages Project", OSI-DS WG document, OSI-DS-39, Dresden University, AIC Systems Laboratory, February 1993.

[5] CCITT Blue Book, "Data Communication Networks: Directory", Recommendations X.500-X.521, December 1988. 